\title{
Understanding Experiences of Moral Distress in End-of-Life Care Among US and UK Physician Trainees: a Comparative Qualitative Study
}

\author{
Sarah Rosenwohl-Mack, MD, MPH' , Daniel Dohan, $P h D^{2}$, Thea Matthews, $B A^{2}$, \\ Jason Neil Batten, $\mathrm{MD}^{3}$, and Elizabeth Dzeng, $\mathrm{MD}, \mathrm{MPH}, \mathrm{PhD}^{2,4}$ (1)
}

'Department of Family Medicine, University of California, San Francisco, San Francisco, CA, USA; ${ }^{2}$ Institute of Health Policy Studies, University of California, San Francisco, San Francisco, CA, USA; ${ }^{3}$ Department of Medicine, Stanford University, Stanford, CA, USA; ${ }^{4}$ Department of Medicine, Division of Hospital Medicine, University of California, San Francisco, San Francisco, CA, USA.

\begin{abstract}
BACKGROUND: Moral distress is a state in which a clinician cannot act in accordance with their ethical beliefs because of external constraints. Physician trainees, who work within rigid hierarchies and who lack clinical experience, are particularly vulnerable to moral distress. We examined the dynamics of physician trainee moral distress in end-of-life care by comparing experiences in two different national cultures and healthcare systems.
\end{abstract}

OBJECTIVE: We investigated cultural factors in the US and the UK that may produce moral distress within their respective healthcare systems, as well as how these factors shape experiences of moral distress among physician trainees.

DESIGN: Semi-structured in-depth qualitative interviews about experiences of end-of-life care and moral distress.

PARTICIPANTS: Sixteen internal medicine residents in the US and fourteen junior doctors in the UK.

APPROACH: The work was analyzed using thematic analysis.

KEY RESULTS: Some drivers of moral distress were similar among US and UK trainees, including delivery of potentially inappropriate treatments, a poorly defined care trajectory, and involvement of multiple teams creating different care expectations. For UK trainees, healthcare team hierarchy was common, whereas for US trainees, pressure from families, a lack of guidelines for withholding inappropriate treatments, and distress around physically harming patients were frequently cited. US trainees described how patient autonomy and a fear of lawsuits contributed to moral distress, whereas UK trainees described how societal expectations around resource allocation mitigated it.

CONCLUSION: This research highlights how the differing experiences of moral distress among US and UK physician trainees are influenced by their countries' healthcare cultures. This research illustrates how experiences of moral distress reflect the broader culture in which it occurs and suggests how trainees may be particularly vulnerable to it. Clinicians and healthcare leaders in both countries can learn from each other about policies and practices that might decrease the moral distress trainees experience.

Prior Presentations $N / A$

Received June 4, 2020

Accepted October 12, 2020

Published online October 27, 2020
KEY WORDS: moral distress; house staff; resident; ethics; end-of-life; trainee.

J Gen Intern Med 36(7):1890-7

DOI: $10.1007 /$ s11606-020-06314-y

(C) Society of General Internal Medicine 2020

\section{INTRODUCTION}

As front-line clinicians, physician trainees are particularly vulnerable to moral distress, which adversely affects both them and their patients. ${ }^{1-4}$ Moral distress occurs when a clinician is unable to act in accordance with their ethical beliefs due to external constraints, particularly those related to hierarchy or institutional culture. ${ }^{5}$ Historically, the topic has been explored primarily among nurses, ${ }^{1,6-8}$ while few studies have been conducted among physicians. ${ }^{2}$ Physician trainees are particularly vulnerable to moral distress as they are frontline clinicians less empowered than attending physicians to make difficult clinical decisions, they carry out potentially distressing resuscitation and procedures, and they typically spend more time with patients and families than attendings. They are also subject to hierarchical and institutional constraints that can make it difficult for them to deliver care consistent with their own ethical and professional values. ${ }^{9}$ Moral distress contributes to burnout ${ }^{3,9}$ as well as to suboptimal patient care. ${ }^{4,10-12}$

End-of-life care is ethically complex, particularly when patients are unable to articulate their preferences for care and surrogates are asked to represent the patient's wishes. Patients or families may desire treatments that clinicians deem medically inappropriate or that they would not wish for themselves, a tension which can also contribute to feelings of distress. Delivering potentially inappropriate high-intensity treatments at the end of life particularly contributes to clinician moral distress. ${ }^{2,3,13-16}$

When comparing the US and the UK, there are institutional and societal differences in how patient autonomy and a patient's best interest are prioritized in end-of-life decision-making. Historically and culturally, the US tends to prioritize patient autonomy, while in contrast, the UK generally 
prioritizes a patient's best interest. ${ }^{17,18}$ In this context, autonomy represents a prioritization of a patient's individual choice and agency, while best interest here refers to actions that adhere to the Beauchamp and Childress principles of beneficence (acting to benefit the patient) and non-maleficence (avoiding actions that harm the patient). ${ }^{19} \mathrm{~A}$ deeper understanding of the way physicians in the US and the UK conceptualize end-of-life care and how divergent ethical prioritization of their health systems informs end-of-life decision-making may help us better contextualize the moral distress that trainees experience.

To our knowledge, no studies have been conducted that characterize experiences of moral distress among physician trainees in the US and the UK. This analysis has two aims: first, to identify cultural and institutional factors in the US and the UK that may produce moral distress within their respective healthcare systems, and second, to compare how these cultural and institutional factors shape experiences of moral distress in providing end-of-life care among physician trainees in the two countries.

\section{METHODS}

\section{Design}

This is an exploratory qualitative study that was explicitly designed to understand the moral distress that physician trainees experience in end-of-life care. This work is also a subset of a larger study investigating clinician practices and perspectives around potentially inappropriate high-intensity treatments in end-of-life care. The study was approved by the IRB of the University of California, San Francisco.

\section{Sample}

Thirty physicians were purposively sampled by stage of training and gender to provide a wide range of perspectives and experiences. We used email list-serves in the US and a snowball sampling strategy in the UK, recruiting residents (US) or junior doctors (UK) in internal medicine or a medicine subspecialty. One researcher (SRM) interviewed 10 UK participants in July-August 2019 and 16 US participants in September-October 2019. Another researcher (ED) conducted 4 UK participant interviews in May 2016 (the temporal gap was due to a lack of funding and personnel), for a total of 16 residents at one US institution and 14 junior doctors at a variety of UK institutions, all of whom completed a process of informed consent. All participants practiced at established academic medical centers. Residents were eligible in the US, and all junior doctors below the level of consultant (including foundation doctors and specialty trainees) were eligible in the UK.

\section{Data Collection}

Researchers conducted semi-structured in-depth interviews with physician trainees in the US and the UK. The initial interview guide was created through a review of the literature and prior research, and revised and adapted throughout the interview process. Theories and hypotheses developed in initial interviews were explored and validated in subsequent ones. Interviews were 40-70 min long. The interviewers asked trainees about their experiences with end-of-life care and decision-making, as well as about their familiarity with the concept of moral distress, and their experiences of moral distress in providing care for patients at the end of life. If they were not familiar with the term "moral distress," it was defined for them. Interviews were conducted in-person, via Zoom video conferencing, or via phone, in accordance with the participant's preference. Interviews were audio-recorded, transcribed verbatim, and anonymized. Data collection and analysis occurred simultaneously throughout the interview process. Data collection was completed when theoretical saturation occurred, such that the same themes recurred and no new themes were generated. ${ }^{20}$

\section{Analysis}

The work was analyzed via thematic analysis. ${ }^{21}$ We used a mixture of inductive and deductive analytic approaches, starting with a related codebook developed by the study team (ED, JNB, TM, DD) in the larger study on clinician attitudes around high-intensity care. We refined and tailored the codebook to this particular study throughout the coding process, adding codes that emerged in the data and were not captured in the existing codebook. Coding occurred in ATLAS.ti. Twenty percent of the interviews were double coded by two researchers, ED (a physician and sociologist) and SRM (a fourth-year medical student), with rare disagreements, which were resolved in discussion. The remainder of the interviews were coded by SRM. After generating initial codes, the team worked together to collate codes into potential themes, prioritizing those that were frequently mentioned. The scope and definition of each theme were refined in reference to the data to inform the results presented here.

\section{RESULTS}

A total of 16 physician trainees participated in the US and 14 in the UK (see Table 1).

Table 1 Participant Characteristics

\begin{tabular}{lll}
\hline \hline & US $(\boldsymbol{n}=\mathbf{1 6})$ & UK $(\boldsymbol{n}=\mathbf{1 4})$ \\
\hline Sex: M, F & 6,10 & 4,10 \\
Age in years: mean (range) & $30(26-39)$ & $30(27-34)$ \\
Years of experience: mean (range) & $2.2(1-3)$ & $3.6(2-6)$ \\
\hline
\end{tabular}

US residency training typically lasts 3-7 years, and UK junior doctor training typically lasts 5-8 years. In the first 2 years of training, UK junior doctors are referred to as foundation doctors $(F 1, F 2)$, after which they work as a specialty registrar in either general practice or a hospital specialty for 3-6 years (ST1-6). 
We identified many factors that participants perceived as contributing to moral distress. These drivers of moral distress function as external constraints that make it difficult or impossible for clinicians to act in accordance with their ethical beliefs. We divide the results in two sections - structural drivers of moral distress that were similar, and those that were different between the US and the UK. Some, such as delivering potentially inappropriate treatments, were similar between US and UK respondents, while others differed, such as disempowerment within the healthcare hierarchy or pressure from families.

\section{STRUCTURAL CONSTRAINTS THAT WERE SIMILAR DRIVERS OF MORAL DISTRESS BETWEEN THE US AND THE UK \\ Potentially Inappropriate Treatments}

Most physician trainees in both the US and the UK felt moral distress around providing treatment they perceived as potentially inappropriate. They commonly described the way treatments prolong suffering and the complicated role families play:

It was clear that she was dying, and we created an option for something that I think was medically inappropriate, but also, there's a tremendous moral component of prolonging suffering in someone, and allowing decisions to be made by families that don't understand that there isn't a better end point. (US, PGY3)

One stated that she felt that the bulk of her job was spent delivering interventions unlikely to have an effect:

I just really think a lot of the job of a [junior doctor] is running around, trying to save people who don't, you're not going to save, doing ABGs [Arterial Blood Gas] and things on people who are going to die anyway. I just feel like it's very futile and quite distressing to everyone, including us, and the nurses, and the patients. (UK, F2)

\section{Poorly Defined Care Trajectory}

Many participants described how terminal patients often initially received all available disease-directed treatments and may progress towards receiving only palliative treatments as their disease progressed. Trainees frequently reported feeling moral distress when treating patients who were receiving neither active treatment nor palliation and whose plan of care was not well defined. This tension is sometimes unavoidable, including times where a patient's disease trajectory is unclear. However, it most often arises when there is a lack of consensus among the patient, family, and clinical team about the type of care a patient should receive.
[There was] a week to two weeks where he was neither actively managed nor was he particularly palliated, and the nursing staff therefore didn't quite know how to treat him, which is a big problem because they spend the most time with him. So, was he being escalated [given more aggressive interventions] in time? Probably not. Were his PRN's [as-needed medications] being given to him properly? Probably not. (UK, ST3)

\section{Involvement of Multiple Teams Creating Different Treatment Expectations}

Some respondents spoke about how, as physicians on the patient's primary treatment team, they felt less able to withhold or withdraw treatments than other teams. One described a code as a procedure, comparing it to other procedures that surgeons can withhold:

One thought that struck me was like the surgeons aren't offering surgery. It's interesting that we don't view like codes [resuscitation] the same way because it is a procedure in a sense. (US, PGY3)

One participant spoke about the way in which different specialties can create different expectations about end-of-life care. She described her experience of oncology specialists helping to de-escalate care:

I think in oncology ward it's much easier to talk to people about decision making and ceilings of care... I guess terminal diagnosis, and people expect to deteriorate and expect it to get worst. So sometimes it's easier to have those conversations. (UK, F2)

Trainees spoke about the distress they experienced when the involvement of multiple teams prolonged an ineffective course of treatment:

Chemotherapy was dangled in front of him as this possible carrot where if you better recover from his pain or did better in the future, he may become eligible for it again ... multiple teams were involved and the fact that the patient really wanted some help perpetuated this very, very long hospitalization where there's always this thought well maybe in a week or in a couple of days chemotherapy would be offered. (US, PGY2)

\section{DIFFERENT STRUCTURAL CONSTRAINTS THAT DRIVE MORAL DISTRESS BETWEEN THE US AND THE UK Disempowerment Within Healthcare Hierarchies}

Many UK respondents described hierarchy as a driver of moral distress, whereas few US trainees did. Some UK 
respondents described difficulty with raising concerns to their consultants (attendings) or suggesting an alternative course of action:

I guess at the end of the day, their names are on the records and everything, and they're worried that we haven't tried everything. Yeah, I've had quite resistant consultants before. (UK, F2)

US participants, by contrast, generally felt empowered to speak up to their attendings if they felt a patient's care was too intensive:

I think when it's explicitly about an end-of-life discussion, I think most of the attendings have been pretty open about at least engaging in a back and forth about that so that I feel like we're both ... I don't think we've ever reached a point where they pull rank and say, no, we're doing this. (US, PGY3)

\section{Pressure from Family}

Most US respondents spoke extensively about the moral distress they experienced when a patient's family advocated for more intensive measures than the physician thought medically appropriate or in a patient's best interest. Relatively few trainee respondents in the UK spoke about distress caused by pressure from families. US respondents spoke about how family members often prioritized their own wishes over those of the patient themselves:

I think that gets tricky, especially when other people become involved. I think I had an ICU attending who called it 'the nephew from Peoria,' the family member who shows up and has not been involved at all and is just like, 'What are you talking about? How could you kill my family member?' (US, PGY3)

Another resident spoke about the challenge of family members who wanted to reverse a patient's "code status," when the patient was incapacitated. "Code status" refers to the level of intervention a patient wants if their heart or breathing stops (e.g., "full code" or "Do Not Resuscitate").

There have been a handful of instances where the family will be like, "This is their POLST [Physician Orders for Life-Sustaining Treatment, a medical document that outlines treatment preferences], and we're reversing the code status," because now it's happening in front of them. I think we always tell patients, "The code status is something that can change at any time," but it's hard I think the first moment when you capture the patient unable to communicate and the family is put in the position. (US, PGY2)

\section{Distress Around Physically Harming Patients}

Many US respondents explicitly referred to the physical trauma that clinicians inflict upon patients in providing aggressive treatments, whereas few respondents in the UK mentioned the topic. US trainee respondents spoke about the way in which the physical labor of performing futile resuscitations caused psychological trauma:

It is so distressing. You are beating someone's body, and often as the medical provider, you personally are beating their body... It is very difficult to go home at the end of the day and be like, "I just did this. I just battered someone today." (US, PGY3)

\section{Systems and Guidelines that Facilitated or Hindered the Ability to Withhold Potentially Inappropriate Treatments}

Many UK participants spoke about guidelines for the use of life-sustaining treatment that made it easier to withdraw or withhold potentially inappropriate treatments:

I think they've got strict criteria [for dialysis] which probably helps... There are certain other things to qualify and if there's quite strict rules it's easier for doctors to say, "Sorry, you don't qualify." (UK, ST2)

In contrast, US respondents described the lack of guidelines for interventions at the end of life. This physician specifically contrasted the system in the US to that in the UK, describing how the individual physician rather than the health system bears more responsibility for making decisions about the limits of treatment at the end of life:

I think it's really hard because as a society we have not really made decisions about how to weigh those against each other. Whereas other societies, for example the National Health Service (NHS) in Britain have made it really clear that certain end-of-life, certain procedures, sort of interventions at the end-of-life will just simply not be covered by the national health care system... So I think as a society we need to kind of have these types of conversations more, so that the burden isn't on an individual [doctor]. (US, PGY3)

\section{ROLE OF SOCIETAL CULTURE}

Most US respondents described how societal culture contributes to moral distress, whereas most in the UK described how societal culture mitigates it.

\section{Patient Autonomy}

Trainees in the US described how the health system's prioritization of patient choice contributed to the difficulty doctors 
face in delivering potentially inappropriate treatments at the end of life:

In other countries for example, people aren't even offered at all and that's just the culture of that country and how they approach end-of-life care. Whereas here we give the decision totally to the family or the patient. (US, PGY3)

\section{Fear of Lawsuits}

Trainees in the US endorsed widespread concern about lawsuits among their colleagues, though nobody reported knowing someone who had been sued for withholding or withdrawing care at the end of life:

I think there's kind of like the liability culture that we live in where sometimes I think people want to be like, "Well, you could do this or this or this," and just putting the burden on the patient, takes the responsibility off the physician. But I don't think that that's actually better for the patient. (US, PGY1)

\section{Resource Allocation}

US trainee respondents spoke rarely about resource allocation in the setting of end-of-life care, whereas many UK respondents spoke explicitly about patient understanding that the NHS had limited resources as a mitigator of moral distress:

I think people are much more understanding than if you were to talk about it in a different healthcare setting... If you explain to people that there's a resource issue, or that things will take time, and they get frustrated, but they understand. (UK, ST2)

\section{DISCUSSION}

Our research highlights the ways US and UK physician trainees experience moral distress differently, informed by their respective countries' healthcare cultures. This research illustrates how experiences of moral distress reflect the broader institutional and social climate in which it occurs and suggests how trainees may be particularly vulnerable to the experience of this distress.

Trainees are particularly vulnerable to moral distress in the delivery of end-of-life care. ${ }^{2,22,23}$ Many causes of moral distress among clinicians are documented in the literature, including providing care not in a patient's best interest, ${ }^{24}$ poor patient care due to lack of continuity or poor communication, ${ }^{25}$ and poor patient care due to time constraints, ${ }^{26}$ but the most common cause described is aggressive and perceived futile treatment, particularly at the end of life. ${ }^{2,13-16}$ Potentially inappropriate high-intensity treatment near the end-of-life is also known to decrease patient quality of life. ${ }^{27,28}$ This study contributes to the literature by identifying and characterizing additional drivers of moral distress that are common or distinct among physician trainees in the US and the UK. By identifying successful practices that are common or accepted in the UK, but not found in the US, we can help identify practices and policies that alleviate moral distress and improve end-oflife care that exists in the UK that could be introduced in the US context.

Several drivers of moral distress described in this study were endorsed by trainees in both the US and the UK. Concern about potentially inappropriate treatments at the end of life is consistent with the existing literature, but the themes of care trajectory and ability to withhold treatment are less well characterized. A patient's care trajectory may be poorly defined for multiple reasons, some of which may be more likely to cause moral distress than others; for example, sometimes a trajectory is poorly defined because of poor communication within a team, whereas other times it is poorly defined because families may need time to come to terms with a loved one's impending death. It is likely that these drivers were consistent between the two countries because they pertain more to the type of treatment given at the end of life than to the systems and structures in which the treatment is given.

Non-beneficial treatment at the end of life is widespread in many countries, ${ }^{29}$ but its prevalence and definition vary based on many factors, including national ${ }^{30-34}$ and institutional culture, ${ }^{35-39}$ which affect clinician decision-making. In the US, a greater degree of aggressive care at the end of life is more often considered appropriate than in the UK.

In terms of medical culture, many UK participants, but few US respondents, noted that hierarchy within the healthcare system contributed to their feelings of moral distress, often in cases in which they felt unable to effectively advocate for less intensive care for a patient. Hierarchy is a commonly reported reason that UK junior doctors do not feel comfortable speaking up in clinical situations, which contributes not only to moral distress but also to medical errors and poor patient safety. ${ }^{40-45}$

We also noted differences between the US and the UK contexts in terms of structures and practices that facilitate withholding potential inappropriate treatments. For US participants, a lack of national or institutional guidelines to stop escalation of potentially inappropriate treatments contributed to moral distress. In contrast, the UK has a robust set of national guidelines that inform end-of-life decision-making, the presence of which helps trainees feel able to resist pressure from families for potentially inappropriate treatment. The UK's National Institute for Health and Care Excellence $(\mathrm{NICE})^{46}$ issues guidance about eligibility for various treatments in a variety of medical settings, and physicians rely on this guidance when offering or withholding treatment at the end of life. ${ }^{47}$ This difference is reflected in different societal and legal norms in the two countries. For example, the UK has significant legal rulings that create public precedents around futile care and limits of acceptable medical treatment, while 
the US lacks similar standards. ${ }^{48}$ In addition, in the UK, the physician, rather than the family, has the ultimate authority to make treatment decisions for patients without decisional capacity at end of life, ${ }^{48-50}$ which may contribute to the decreased pressure UK trainees endorsed feeling from families. In the US, the framework of substituted judgment places the decision-making for a patient without capacity in the hands of the family. ${ }^{51}$

Finally, broader societal and cultural norms in US and UK appear to affect moral distress. In this study, UK physicians described how a common understanding about the importance of resource allocation helped physicians and families reach an understanding about the appropriate treatment for a patient at the end of life. This likely reflects the strong public image of the NHS as a shared but limited resource. In the US, by contrast, a mentality of consumerism and individual choice drives highintensity care. ${ }^{52} \mathrm{We}$ found that increased family pressure contributed to moral distress experienced by US participants. This increased family pressure in the US relative to the UK is likely attributable at least in part to the different healthcare expectations that patients have in each country. ${ }^{52}$ In addition, prioritization of patient autonomy and a fear of lawsuits further contributed to the moral distress US trainees experience in trying to avoid potentially inappropriate treatments.

Some research exists on ways to combat physician moral distress, including wider adoption of Schwartz Rounds, which focus on the shared humanity of both patients and clinicians. ${ }^{53,54}$ Other options include the implementation of specific clinical practice frameworks and educational programs aiming to help clinicians reframe the ways in which they think about distressing situations. ${ }^{55-57}$ This study also presents opportunities for clinicians in both countries to learn from each other about policies and practices that might decrease the moral distress trainees experience, while also decreasing potentially inappropriate treatments at the end of life. For example, by adopting more guidelines for defining appropriate care at the end of life, US hospitals could enable their residents, with proper supervision, to more easily withhold or withdraw potentially inappropriate treatments at the end of life. UK hospitals could work to decrease the role of hierarchy, especially around end-of-life decision-making, such that UK trainees feel more empowered to speak up if they disagree with a patient's treatment plan. This research underscores the importance of further understanding the impact of potentially inappropriate end-of-life treatments on moral distress and creating guidelines around the provision of appropriate endof-life treatments to address both quality of end-of-life care and physician moral distress. ${ }^{3}$

Limitations of this study include social desirability bias in interviews with physician trainees. In addition, we only interviewed trainees at academic medical programs, which may affect the types of clinical cases that trainees encounter and may also have more structures like Morbidity and Mortality Rounds, conferences, or other opportunities to reflect on their experiences of moral distress. In addition, participants came from one site in the US and multiple sites in the UK. This limitation is partially mitigated by the fact that this study investigated moral distress experienced by the individual physician and did not seek explicitly to understand factors affecting moral distress that varied by institution.

A focus on clinician moral distress is even more important now in the setting of the COVID-19 pandemic. Given a growing scarcity of resources, the pandemic creates a setting in which explicitly rationing care-whether personal protective equipment, hospital beds, or ventilators-becomes necessary, prompting ethical dilemmas and inevitable moral distress. Other aspects of pandemic response, including clinician burnout, infection of healthcare workers, and witnessing increased sickness and death, can all also contribute to moral distress. As we address the physical health consequences and public health ramifications of the pandemic, we must also pay keen attention to the moral distress clinicians experience in these times. Developing clear ethical guidelines for allocation of scarce resources ${ }^{58}$ and robust strategies to mitigate moral distress $^{59}$ in the setting of this pandemic is a crucial first step.

Acknowledgments: We would like to thank the physician trainees who participated in this study.

Corresponding Author: Elizabeth Dzeng, MD, MPH, PhD; Department of Medicine, Division of Hospital Medicine, University of California, San Francisco, San Francisco, CA, USA (e-mail: liz. dzeng@ucsf.edu).

Author Contributions ED and SRM contributed to the conception, study design, creation of the interview guide, and data collection for the study. SRM and ED analysed the data and SRM, ED, and DD interpreted the data. SRM and ED drafted the manuscript, and SRM, $E D, D D, J B N$, and TM revised the manuscript. All authors vouch for the accuracy and completeness of the manuscript and reviewed and approved the final manuscript.

Funding This study was funded through the following sources: UCSF Gibbs Family Medicine Fellowship, California Department of Public Health Alzheimer's Disease Research Award, NIA Grants for Early Medical/Surgical Subspecialty Transition to Aging Research (GEMSSTAR) (1R03AG060098).

\section{Compliance with Ethical Standards:}

Disclaimer: The content is solely the responsibility of the authors and does not necessarily represent the official views of the NIH.

Conflict of Interest: The authors declare that they do not have a conflict of interest.

\section{REFERENCES}

1. Morley G, Ives J, Bradbury-Jones C, Irvine F. What is 'moral distress'? A narrative synthesis of the literature: Nurs Ethics. Published online October 8, 2017. https://doi.org/10.1177/0969733017724354

2. Dzeng E, Colaianni A, Roland M, et al. Moral Distress Amongst American Physician Trainees Regarding Futile Treatments at the End of Life: A Qualitative Study. J Gen Intern Med 2016;31(1):93-99. https:// doi.org/10.1007/s11606-015-3505-1

3. Dzeng E, Curtis JR. Understanding ethical climate, moral distress, and burnout: a novel tool and a conceptual framework. BMJ Qual Saf 2018;27(10):766-770. https://doi.org/10.1136/bmjqs-2018-007905 
4. Dewa CS, Loong D, Bonato S, Trojanowski $\mathbf{L}$. The relationship between physician burnout and quality of healthcare in terms of safety and acceptability: a systematic review. BMJ Open. 2017;7(6). https://doi.org/ 10.1136/bmjopen-2016-015141

5. Jameton A. Nursing Practice: The Ethical Issues. eweb:51336. Published 1984. Accessed January 17, 2020. https://repository.library.georgetown. edu/handle/10822/800986

6. Jameton A. What Moral Distress in Nursing History Could Suggest about the Future of Health Care. AMA J Ethics 2017;19(6):617-628. https://doi.org/10.1001/journalofethics.2017.19.6.mhst1-1706

7. McCarthy J, Gastmans C. Moral distress: a review of the argumentbased nursing ethics literature. Nurs Ethics 2015;22(1):131-152. https://doi.org/10.1177/0969733014557139

8. Rainer J, Schneider JK, Lorenz RA. Ethical dilemmas in nursing: An integrative review. J Clin Nurs 2018;27(19-20):3446-3461. https://doi. org/10.1111/jocn. 14542

9. Dzeng E, Wachter RM. Ethics in Conflict: Moral Distress as a Root Cause of Burnout. J Gen Intern Med. 35:409 Published online October 30, 2019. https://doi.org/10.1007/s11606-019-05505-6

10. Dzau VJ, Kirch DG, Nasca TJ. To Care Is Human - Collectively Confronting the Clinician-Burnout Crisis. N Engl J Med. 378:312 Published online January 24, 2018. https://doi.org/10.1056/ NEJMp 1715127

11. Salyers MP, Bonfils KA, Luther L, et al. The Relationship Between Professional Burnout and Quality and Safety in Healthcare: A MetaAnalysis. J Gen Intern Med 2017;32(4):475-482. https://doi.org/10. 1007/s11606-016-3886-9

12. Panagioti $\mathbf{M}$, Geraghty $\mathbf{K}$, Johnson $\mathbf{J}$, et al. Association Between Physician Burnout and Patient Safety, Professionalism, and Patient Satisfaction: A Systematic Review and Meta-analysis. JAMA Intern Med 2018;178(10):1317-1331. https://doi.org/10.1001/jamainternmed. 2018.3713

13. Lusignani M, Giannì ML, Re LG, Buffon ML. Moral distress among nurses in medical, surgical and intensive-care units. $J$ Nurs Manag 2017;25(6):477-485. https://doi.org/10.1111/jonm.12431

14. Hamric AB, Blackhall LJ. Nurse-physician perspectives on the care of dying patients in intensive care units: collaboration, moral distress, and ethical climate. Crit Care Med 2007;35(2):422-429. https://doi.org/10. 1097/01.CCM.0000254722.50608.2D

15. Ferrell BR. Understanding the moral distress of nurses witnessing medically futile care. Oncol Nurs Forum 2006;33(5):922-930. https:// doi.org/10.1188/06.ONF.922-930

16. Mobley MJ, Rady MY, Verheijde JL, Patel B, Larson JS. The relationship between moral distress and perception of futile care in the critical care unit. Intensive Crit Care Nurs 2007;23(5):256-263. https:// doi.org/10.1016/j.iccn.2007.03.011

17. Bishop JP, Brothers KB, Perry JE, Ahmad A. Reviving the conversation around CPR/DNR. Am J Bioeth 2010;10(1):61-67. https://doi.org/10. $1080 / 15265160903469328$

18. Paris JJ, Ahluwalia J, Cummings BM, Moreland MP, Wilkinson DJ. The Charlie Gard case: British and American approaches to court resolution of disputes over medical decisions. $J$ Perinatol 2017;37(12):1268-1271. https://doi.org/10.1038/jp.2017.138

19. Beauchamp TL, Childress JF. Principles of Biomedical Ethics. 7. Oxford University Press; Oxford 2012

20. Guest G, Bunce A, Johnson L. How Many Interviews Are Enough?: An Experiment with Data Saturation and Variability. Field Methods. 18:59 Published online 2006. https://doi.org/10.1177/1525822X05279903

21. Braun V, Clarke V. Using thematic analysis in psychology. Qual Res Psychol 2006;3(2):77-101. https://doi.org/10.1191/ 1478088706qp063oa

22. Rosenthal MS, Clay M. Initiatives for Responding to Medical Trainees' Moral Distress about End-of-Life Cases. AMA J Ethics 2017;19(6):585594. https://doi.org/10.1001/journalofethics.2017.19.6.stas1-1706

23. Winkenwerder W. Ethical Dilemmas for House Staff Physicians. J Am Med Assoc 1985;254(24):3454-3457.

24. Hamric AB, Borchers CT, Epstein EG. Development and Testing of an Instrument to Measure Moral Distress in Healthcare Professionals. AJOB Prim Res 2012;3(2):1-9. https://doi.org/10.1080/21507716.2011. 652337

25. Whitehead PB, Herbertson RK, Hamric AB, Epstein EG, Fisher JM. Moral distress among healthcare professionals: report of an institutionwide survey. J Nurs Scholarsh 2015;47(2):117-125. https://doi.org/10. $1111 /$ jnu. 12115

26. Astbury JL, Gallagher CT. Moral distress among community pharmacists: causes and achievable remedies. Res Soc Adm Pharm. 16:321
Published online May 29, 2019. https://doi.org/10.1016/j.sapharm. 2019.05.019

27. Wright AA, Keating NL, Ayanian JZ, et al. Family Perspectives on Aggressive Cancer Care Near the End of Life. JAMA. 2016;315(3):284292. https://doi.org/10.1001/jama.2015.18604

28. Prigerson HG, Bao Y, Shah MA, et al. Chemotherapy Use, Performance Status, and Quality of Life at the End of Life. JAMA Oncol 2015;1(6):778784. https://doi.org/10.1001/jamaoncol.2015.2378

29. Cardona-Morrell M, Kim JCH, Turner RM, Anstey M, Mitchell IA, Hillman K. Non-beneficial treatments in hospital at the end of life: a systematic review on extent of the problem. Int $J$ Qual Health Care 2016;28(4):456-469. https://doi.org/10.1093/intqhc/mzw060

30. Sprung CL, Truog RD, Curtis JR, et al. Seeking Worldwide Professional Consensus on the Principles of End-of-Life Care for the Critically Ill. The Consensus for Worldwide End-of-Life Practice for Patients in Intensive Care Units (WELPICUS) Study. Am J Respir Crit Care Med 2014;190(8):855-866. https://doi.org/10.1164/rccm.201403-0593CC

31. Cohen S, Sprung $\mathbf{C}$, Sjokvist $\mathbf{P}$, et al. Communication of end-of-life decisions in European intensive care units. Intensive Care Med 2005;31(9): 1215-1221. https://doi.org/10.1007/s00134-005-2742-x

32. Mobeireek AF, Al-Kassimi F, Al-Zahrani K, et al. Information disclosure and decision-making: the Middle East versus the Far East and the West. $J$ Med Ethics 2008;34(4):225-229. https://doi.org/10.1136/jme.2006. 019638

33. Lobo SM, De Simoni FHB, Jakob SM, et al. Decision-Making on Withholding or Withdrawing Life Support in the ICU: A Worldwide Perspective. Chest 2017;152(2):321-329. https://doi.org/10.1016/j. chest.2017.04.176

34. Boivin A, Marcoux I, Garnon G, et al. Comparing end-of-life practices in different policy contexts: a scoping review. $J$ Health Serv Res Policy 2015;20(2):115-123. https://doi.org/10.1177/1355819614567743

35. Dzeng E, Colaianni A, Roland M, et al. Influence of institutional culture and policies on do-not-resuscitate decision making at the end of life. JAMA Intern Med 2015;175(5):812-819. https://doi.org/10.1001/ jamainternmed.2015.0295

36. Barnato AE, Tate JA, Rodriguez KL, Zickmund SL, Arnold RM. Norms of decision making in the ICU: a case study of two academic medical centers at the extremes of end-of-life treatment intensity. Intensive Care Med 2012;38(11):1886-1896. https://doi.org/10.1007/s00134-0122661-6

37. Barnato AE, Mohan D, Lane RK, et al. Advance care planning norms may contribute to hospital variation in end-of-life ICU use: a simulation study. Med Decis Making 2014;34(4):473-484. https://doi.org/10.1177/ 0272989X14522099

38. Quill CM, Ratcliffe SJ, Harhay MO, Halpern SD. Variation in decisions to forgo life-sustaining therapies in US ICUs. Chest 2014; 146(3):573-582. https://doi.org/10.1378/chest.13-2529

39. Wunsch H, Harrison DA, Harvey S, Rowan K. End-of-life decisions: a cohort study of the withdrawal of all active treatment in intensive care units in the United Kingdom. Intensive Care Med 2005;31(6):823-831. https://doi.org/10.1007/s00134-005-2644-y

40. Vivekananda-Schmidt P, Vernon B. FY1 doctors' ethicolegal challenges in their first year of clinical practice: an interview study. $J$ Med Ethics 2014;40(4):277-281. https://doi.org/10.1136/medethics-2011-100391

41. Ross S, Ryan C, Duncan EM, et al. Perceived causes of prescribing errors by junior doctors in hospital inpatients: a study from the PROTECT programme. BMJ Qual Saf 2013;22(2):97-102. https://doi. org/10.1136/bmjqs-2012-001175

42. Tallentire VR, Smith SE, Skinner J, Cameron HS. Understanding the behaviour of newly qualified doctors in acute care contexts. Med Educ 2011;45(10):995-1005. https://doi.org/10.1111/j.1365-2923.2011. 04024.x

43. Mattick K, Kelly N, Rees C. A window into the lives of junior doctors: narrative interviews exploring antimicrobial prescribing experiences. $J$ Antimicrob Chemother 2014;69(8):2274-2283. https://doi.org/10.1093/ jac/dku093

44. Hooper P, Kocman D, Carr S, Tarrant C. Junior doctors' views on reporting concerns about patient safety: a qualitative study. Postgrad Med J 2015;91(1075):251-256. https://doi.org/10.1136/postgradmedj-2014133045

45. Bradley V, Liddle S, Shaw R, et al. Sticks and stones: investigating rude, dismissive and aggressive communication between doctors. Clin Med Lond Engl 2015;15(6):541-545. https://doi.org/10.7861/clinmedicine. 15-6-541 
46. National Institute for Health and Care Excellence. NICE guidance. NICE. Published 2020. Accessed January 26, 2020. https://www.nice.org.uk/ guidance

47. National Institute for Health and Care Excellence. End of life care | Topic | NICE. Published 2020. Accessed January 26, 2020. https://www.nice. org.uk/guidance/health-and-social-care-delivery/end-of-life-care

48. Bosslet GT, Baker M, Pope TM. Reason-Giving and Medical Futility: Contrasting Legal and Social Discourse in the United States With the United Kingdom and Ontario, Canada. Chest. 2016;150(3):714-721. https://doi.org/10.1016/j.chest.2016.05.026

49. National Health Service. National Health Service. NHS East of England Integrated Do Not Attempt Cardiopulmonary Resuscitation (DNACPR)—Policy for Adults. Published online 2011. https://heeoe.hee. nhs.uk/sites/default/files/dnacpr_policy_-_east_of_england_1.pdf. Accessed on Oct 31, 2012

50. General Medical Council. Response to concerns about our guidance for doctors on attempting CPR and DNACPR orders. Published 2017. Accessed January 18, 2020. https://www.gmc-uk.org/news/news-archive/response-to-concerns-about-our-guidance-for-doctors-onattempting-cpr-and-dnacpr-orders

51. Vig EK, Taylor JS, Starks H, Hopley EK, Fryer-Edwards K. Beyond substituted judgment: How surrogates navigate end-of-life decisionmaking. J Am Geriatr Soc 2006;54(11):1688-1693. https://doi.org/10. $1111 / j .1532-5415.2006 .00911 . x$

52. Starr P. The social transformation of American medicine: The rise of a sovereign profession and the making of a vast industry. New York. Basic Books; 1982.
53. Penson RT, Schapira L, Mack S, Stanzler M, Lynch TJ. Connection: Schwartz Center Rounds at Massachusetts General Hospital Cancer Center. Oncologist 2010;15(7):760-764. https://doi.org/10.1634/ theoncologist.2009-0329

54. Schwartz Rounds. The Schwartz Center. Accessed February 19, 2020. https://www.theschwartzcenter.org/programs/schwartz-rounds

55. Rushton CH, Kaszniak AW, Halifax JS. Addressing moral distress: application of a framework to palliative care practice. $J$ Palliat Med 2013;16(9): 1080-1088. https://doi.org/10.1089/jpm.2013.0105

56. Back AL, Rushton CH, Kaszniak AW, Halifax JS. "Why are we doing this?": clinician helplessness in the face of suffering. $J$ Palliat Med 2015; 18(1):26-30. https://doi.org/10.1089/jpm.2014.0115

57. Krasner MS, Epstein RM, Beckman H, et al. Association of an educational program in mindful communication with burnout, empathy, and attitudes among primary care physicians. JAMA. 2009;302(12):1284-1293. https://doi.org/10.1001/jama.2009.1384

58. Emanuel EJ, Persad G, Upshur R, et al. Fair Allocation of Scarce Medical Resources in the Time of Covid-19. N Engl J Med. 2020;382(21):2049. https://doi.org/10.1056/NEJMsb2005114

59. COVID-19: Supporting Ethical Care and Responding to Moral Distress in a Public Health Emergency. The Hastings Center. Accessed March 26, 2020 . https: / / w w w. the hastingscenter.org/ guidancetoolsresourcescovid19/

Publisher's Note: Springer Nature remains neutral with regard to jurisdictional claims in published maps and institutional affiliations. 